\title{
The Fabrication and Function Evaluation of 3D LSPR Biosensor by using CNTs/Au Nanoparticles
}

\author{
Ryohei Fujieda and Yang Ming
}

\begin{abstract}
In the conventional sensor using localized surface Plasmon resonance (LSPR), metallic particle has been only allocated on substrate in two dimensions. We attempt to allocate metal particles in three dimensions to improve sensitivity than a conventional LSPR sensor. As study process, CNTs were synthesized on the Si substrate as support by the CVD method, and modifying gold nanoparticles on surface of CNTs by the PVD method. Evaluation of applicability to bio-sensor was carried out by using protein adsorption. BSA as a protein was applied to the adsorption test. To improve the sensitivity of LSPR, CNTs was patterned by the lithography. Then structural size (Diameter and Pitch) was changed by lithography. For establishment of a new LSPR sensor, we design the optimal structure of detection of low concentration antigen. After BSA adhesion, narrower diameter and pitch of patterned CNTs, longer shift amount was confirmed. And, when Au deposition angle changed $(\theta: 0 \rightarrow 45)$, shift amount was increased. Therefore, we were able to confirm that the substrate which was allocated metal particles in three dimensions had the function as the sensor, and had a potential to improve LSPR sensitivity by optimal design of CNTs patterning.
\end{abstract}

Index Terms—LSPR, Biosensor, CNTs, nanoparticles.

\section{INTRODUCTION}

In recent years, to prevent expanding of the infectious disease such as influenza, the development of biosensor that is rapid and simple is demanded. There is an advantage of real time measurement, label free and miniaturization of measurement device in localized surface plasmon resonance (LSPR). It is paid attention from medicine to biochemistry by these advantages in a wide field. It is applied as an instrument for analysis of high performance now. It will be applied as a sensor in the future, and there is a possibility that SPR spreads widely. As principle of SPR, Free electron on surface of the particle is excited when light irradiates the metal microparticle on the substrate, and a rapid optical absorption happens in a certain wavelength area because plasmon couples it with a light electric field. The wavelength area of the optical absorption shifts by the permittivity change around particle when the antigen such as viruses adhere antibody on this metal microparticle, therefore the application as the sensor is expected. However, it is difficult to detect the antigen of the low concentration and the concentration region that can be detected is narrow,

Manuscript received December 19, 2011; revised January 17, 2012.

This work was supported in part by Tokyo Govement under Grant.

R. Fujieda is with Department of System Design, Tokyo MetropolitanUniversity,191-0065,Tokyo,Japan(e-mail:fujieda-ryouhei@sd. tmu.ac.jp).

M. Yang is with Department of System Design, Tokyo Metropolitan University, 191-0065,Tokyo,Japan(e-mail: yang@tmu.ac.jp). because in the conventional sensor using localized surface Plasmonresonance, the metallic particle has only allocated on substrate in two dimensions [1]- [4].

In addition, gold nanoparticles are widely investigated in many applications because their physical and chemical properties are dependent on their size, shape, composition and morphology. Recently, controlling the aggregation of nanoparticles has attracted a great deal of attention due to their unique structural and optical properties [5-6]. Some properties that are distinctly different from those of the corresponding isolated nanoparticles often emerge from particle aggregation. For example, greatly enhanced third-order nonlinear responses from twin-linked gold nanoparticles (TGNPs) with silica thin coating self-assembled on a glass substrate have been observed [7]. Moreover, the localized surface plasmon resonance (LSPR) can be tuned by controlling the aggregation of gold nanoparticles.

In this study, we attempt to allocate metal particles in three dimensions by using support to improve sensitivity than a conventional LSPR sensor, and aim at two points, one is the establishment of a new LSPR sensor and the other is optimal design of detection of low concentration antigen. Arrays of the particles are enabled by growing carbon nanotubes (CNTs) to reaction field, so we used CNTs as a support and modifying the metal microparticles on the surface of CNTs. CNTs are a tube-shaped material with diameter from several to several hundred $\mathrm{nm}$.

CNTs have been attracting significant attention because of their unique characteristics [8], [9]. Due to their excellent properties, CNTs are studied in various fields [10]- [12]. Using CNTs in sensor device is one of them. Because its high-aspect ratio structure and high specific surface structure.

\section{EXPERIMENT}

\section{A. Approach}

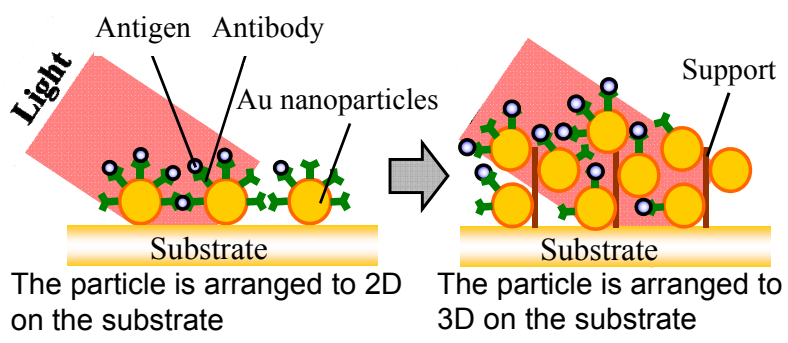

Fig. 1. Competision of allanged Au nanoperticles in 2-dimentions and 3dimentions.

In this study, to improve sensitivity of LSPR sensor, we 
arranged the $\mathrm{Au}$ nanoparticle to three dimensions on substrate by using supports. We used carbon nanotubes as a supports. Fig. 1 shows competision of allanged $\mathrm{Au}$ nanoparticles in 2-dimentions and 3-dimentions. By arranging $\mathrm{Au}$ nanoparticle to three dimensions, the number of Au nanoparticles which light is irradiated increase. As a result, strength of LSPR can be increased, and more sensitive sensor than conventional one can be fabricated.

\section{B. Fabrication of CNTs/Au Nanoparticles Substrate}

As CNTs systhesis method, CNTs was synthesized by chemical vapor deposition (CVD) method. Aluminum as catalyst adjuvant and iron as catalyst was deposited on $\mathrm{Si}$ substrate with the oxide film by Electron Cyclotron Resonance (ECR) -type ion shower device, and the substrate was set up in vacuum chamber. The carbon gas which assumed alcohol raw materials was filled in the chamber, and the temperature of the substrate is heated to about $700^{\circ} \mathrm{C}$. 0As method of $\mathrm{Au}$ nanoparticles deposition on CNTs, Au nanoparticles were deposited by ion coater, and as method of CNT clumping, CNTs was clumped by dropping ethanol $(10 \mu \mathrm{l})$ on the substrate and dried. This substrate was used by investigation of LSPR characteristic.

\section{Experiment Procedure}

Fig. 2 shows experimental process. First, we attempted LSPR analysis of the sample as it received. Second, we attempted LSPR analysis of the sample which was filled in the phosphate buffer as solvent of the protein and after dry. Third, we attempted LSPR analysis of the sample which was filled in $100 \mathrm{mg} / \mathrm{l}$ bovine serum albumin (BSA) and after dry.

Fig. 3 shows experimental method. white light is irradiated to the all substrate, $\mathrm{P}$ wave and Swave of reflected light is taken into detector respectively by polarizer, and the light intensity spectrum is obtained. Fundamentally, the irradiation angle was set as 30 degrees.

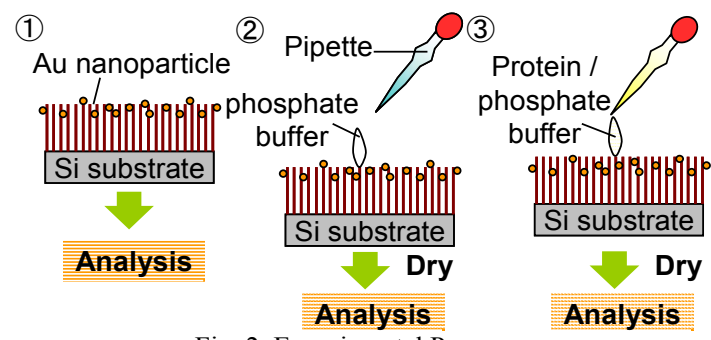

Fig. 2. Experimental Process.

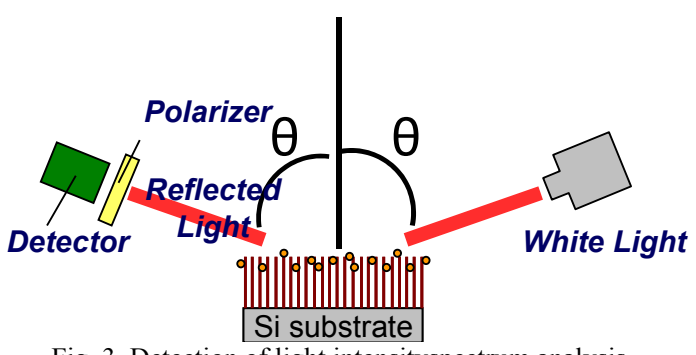

Fig. 3. Detection of light intensityspectrum analysis.

First, Au deposition time was set as 30 s by ion coater. We investigated infulence of $\mathrm{Au}$ deposition time changing $(30 \mathrm{~s}, 60 \mathrm{~s}, 120 \mathrm{~s})$ in our previous study[13], then when $\mathrm{Au}$ deposition time in 60s and 120s. Au nanoparticles on CNTs formed like a thin film, so we decided that $\mathrm{Au}$ deposition time was 30s. In addition, it is desired that the distance between $\mathrm{Au}$ nanoparticles is all constant to improve the sensitivity of LSPR, so CNTs was patterned by the lithography process as shown in Fig.4. Pattern structure size (Diameter and Pitch) was 4 types ; Type A(D:10um, $\mathrm{P}: 20 \mathrm{um})$, Type B(D:10um, P:15um), Type C(D:5um, P:10um), Type D(D:5um, P:7um).

The $\mathrm{Au}$ nanoparticles were modified on the patterned CNTs by ion shower device and it was clumped by the ethanol. The LSPR characteristic was evaluated and the samples were observed by SEM.

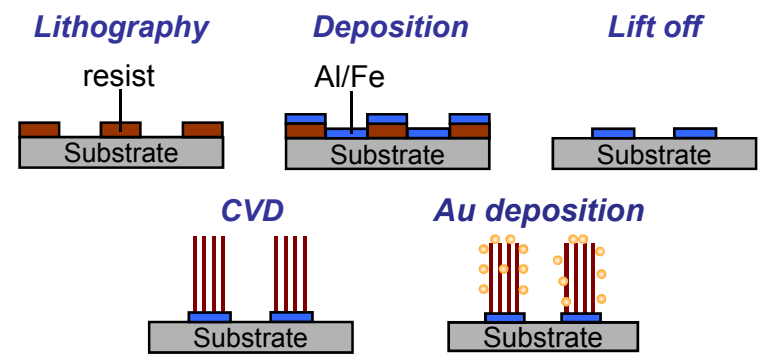

Fig. 4. Lithography process for CNTs patterning.

\section{Evaluation of Changing Au Deposition Method}

Next, we evaluated the influence which changing $\mathrm{Au}$ deposition method has on LSPR characteristic, so we changed Au nanoparticles deposition system (Au deposition angle $\theta: 0 \rightarrow 45$ ).Fig. 5 shows $\mathrm{Au}$ deposition methods. In previous method, $\mathrm{Au}$ deposition angle was $0^{\circ}$, and new method, it was $45^{\circ}$. Total Au coating time was same. In this experiment, we used BSA as a protein; this concentration was $100 \mathrm{mg} / \mathrm{l}$.

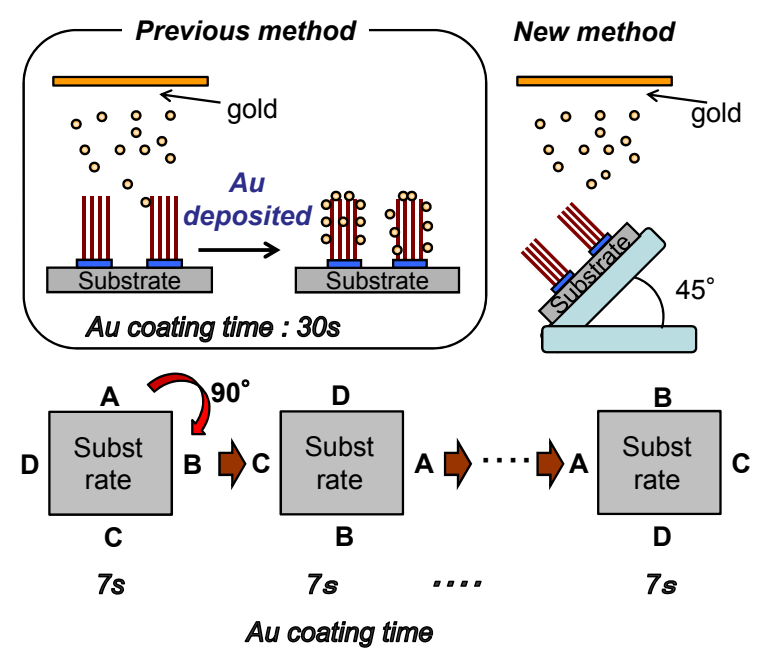

Fig. 5. Au deposition methods (Method 1: $\theta=0$ Method $2: \theta=45)$.

\section{E. Evaluation of Changing Irradiation/Detection Angle}

We evaluated the influence which changing irradiation/detection angle of light has on LSPR characteristic, so we changed these angles: 30,40 , and 45 degree. Fig. 6 shows chang of irradiation/ detection angle . In this experiment, we used BSA as a protein; this concentration was $100 \mathrm{mg} / 1$, and patterned CNTs used this experiment was CNTs which diameter has $5 \mu \mathrm{m}$, and Pitch has $10 \mu \mathrm{m}$. 


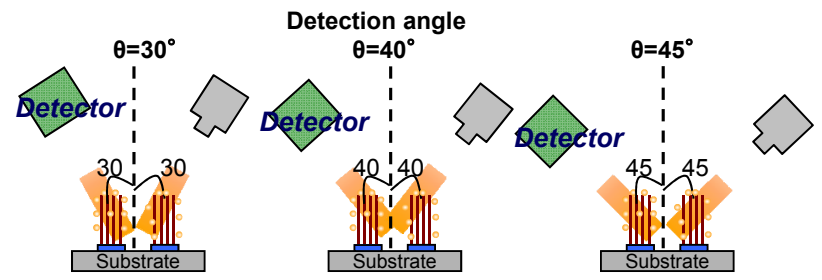

Fig. 6. Change of irradiation/detection angle.

\section{F. Evaluation of Detection Limit}

We evaluated detection method of this 3-dimentional LSPR sensor. We used BSA as a protein, these concentrations were $1 \mathrm{ng} / \mathrm{ml}, 1,10,100 \mathrm{ug} / \mathrm{ml}$. By using these BSA concentrations, we evaluated LSPR characteristic before and after adherence of BSA, and The relation between a shift amount and concentration was shown.

\section{RESULT AND DISCUSSION}

\section{A. LSPR Characteristic of Patterned CNTs}

We synthesized CNTs on substrate by CVD method at first. However, aggregation structure of CNTs was not always constant even if CNTs substrates which have same length are clumped. Hence, there is no repeatability in the shift amount of maximum adsorption wavelength after protein attachments. In addition, it is desired that the distance between Au nanoparticles is all constant to improve the sensitivity of LSPR, so we tried to fabricate patterned CNTs by the lithography. Fig.7 shows surface figure of patterned CNTs. It can be confirmed that CNTs was aligned well by the lithography. The length of CNTs was all $3 \mu \mathrm{m}$, in patterned CNTs substrates respectively. A', B', C', D' was SEM image after CNTs clumping by the ethanol.

In all substrate, Au nanoparticles were deposited by ion coater. The Au deposition methods have two kinds, one was $\mathrm{Au}$ deposition angle in 0 degree, and the other was it in 45 degree.

Fig. 8 shows SEM images in two Au deposition methods. These SEM images show patterned CNTs (Diameter: 5um, Pitch: 10um), Au deposition angles were $0^{\circ}$ in (A), and $45^{\circ}$ in (B). When the deposition angle was 0 degree, $\mathrm{Au}$ nanoparticle has deposited on the upper surface of CNTs. On the other hand, when the deposition angle was 45 degrees, Au nanoparticle has deposited on both the upper and the side of CNT surface.

Fig. 9 shows LSPR characteristic of changing $\mathrm{Au}$ deposition angle. BSA concentration was $100 \mathrm{mg} / \mathrm{ml}$. In Au deposition angle $(\theta=0)$, absorption peak of patterned CNTs (D: $10 \mu \mathrm{m}, \mathrm{P}: 20 \mu \mathrm{m})$ before BSA attachment was $639.6 \mathrm{~nm}$, and it after BSA attachment was $640.4 \mathrm{~nm}$. In Au deposition angle $(\theta=45)$, it before BSA attachment was $640.84 \mathrm{~nm}$, and it after BSA attachment was $644.84 \mathrm{~nm}$. Similarly, In Au deposition angle $(\theta=0)$, absorption peak of patterned CNTs (D: $10 \mu \mathrm{m}, \mathrm{P}: 15 \mu \mathrm{m})$ was from 647.12 to $650.76 \mathrm{~nm}$ after BSA attachment, in Au deposition angle $(\theta=45)$, it was from 643.51 to $655.47 \mathrm{~nm}$. And, In Au deposition angle $(\theta=$ 0 ), absorption peak of patterned CNTs (D: $5 \mu \mathrm{m}, \mathrm{P}: 10 \mu \mathrm{m})$ was from 641.95 to $647.72 \mathrm{~nm}$ after BSA attachment, in $\mathrm{Au}$ deposition angle $(\theta=45)$, it was from 651.04 to $664.96 \mathrm{~nm}$.

After BSA adhesion, more narrow diameter and pitch of patternd CNTs, shift amount was more increase. And, when $\mathrm{Au}$ deposition angle changed $(\theta: 0 \rightarrow 45)$, shift amount was increased. And, when Au deposition angle changed $(\theta: 0 \rightarrow$ 45), shift amount was increased. Adsorption intensity has changed after BSA adhesion because absorbing ability of CNTs weakened when BSA adhered to CNTs too.

Fig. 10 shows shift amount and kinds of substrate in LSPR characteristic of changing $\mathrm{Au}$ deposition angle. Especially, the shift amount of patterned CNTs (D: $5 \mu \mathrm{m}, \mathrm{P}$ : $10 \mu \mathrm{m})$ was biggest in all substrate, it was about $14 \mathrm{~nm}$.

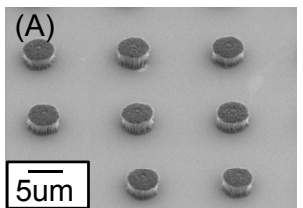

(a)

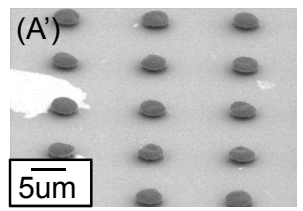

(a')

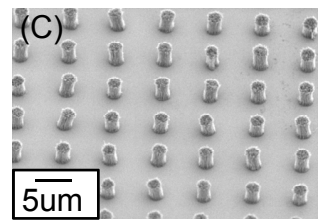

(c)

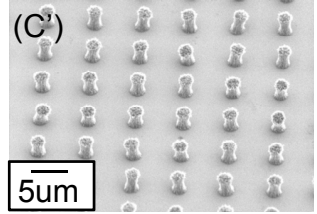

(c')

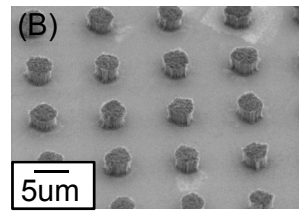

(b)

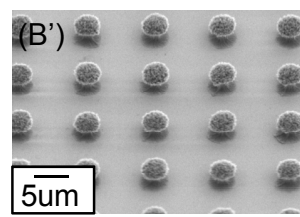

(b’)

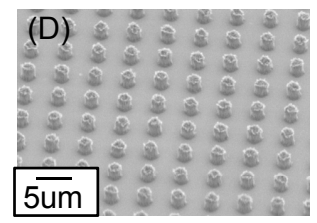

(d)

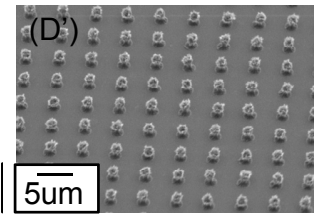

(d')

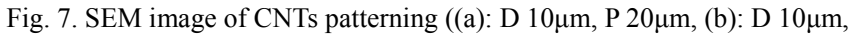
P $15 \mu \mathrm{m},(\mathrm{c}):$ D $5 \mu \mathrm{m}, \mathrm{P} 10 \mu \mathrm{m} \mathrm{D},(\mathrm{d}):$ D $5 \mu \mathrm{m}, \mathrm{P} 7 \mu \mathrm{m})$.

When Au nanoparticles deposition method changed, the number of Au nanoparticles on CNTs surface increases, so it increased the total absorption of excitation light. Futheremore, by reducing the diameter

and pitch, the efficiency of plasmon resonance increased. It is thought that the shift amount to the infrared region can be improved by reducing the interval of CNTs (pitch of the mask).

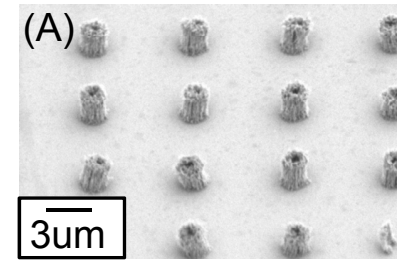

(a)

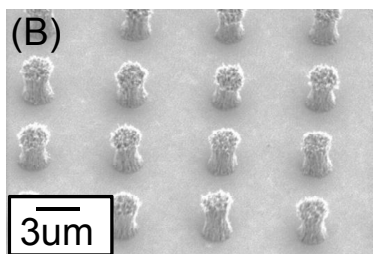

(b)
Fig. 8. Patterned CNTs (D: $5 \mu \mathrm{m}, \mathrm{P}: 10 \mu \mathrm{m}$ ) in Au deposition angle (a) $0^{\circ}$, and (b) $45^{\circ}$.

When a deposition angle was 45 degrees, the shift amount increased compared with the deposition angle of 30 degrees. Because $\mathrm{Au}$ nanoparticle were deposited on the side of $\mathrm{CNTs}$, the optical absorption power of $\mathrm{Au}$ nanoparticle 
increases and it is considered that dependence of the refractive-index change by a protein accretion became large. Furthermore, when the pitch and diameter of CNTs became small, the shift amount increased. This is that a pitch and a diameter become small, and since the distance between $\mathrm{Au}$ nanoparticles became small, it is considered that the plasmon resonance between particles became strong. Moreover, since the CNTs structure per unit area increases and $\mathrm{Au}$ nanoparticle also increases because a pitch and a diameter become small, so it is thought that the whole optical absorption power became strong.

\section{Au coating angle $\left(\theta=0^{\circ}\right) \quad$ Au coating angle $\left(\theta=45^{\circ}\right)$}

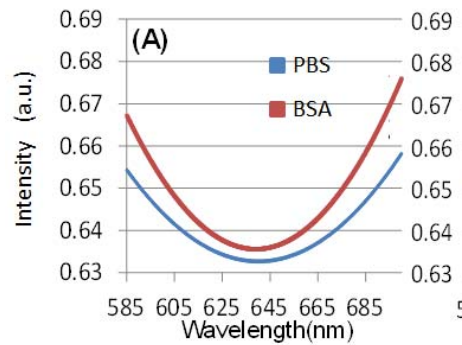

(a)

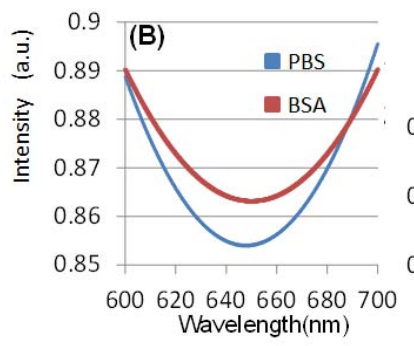

(b)

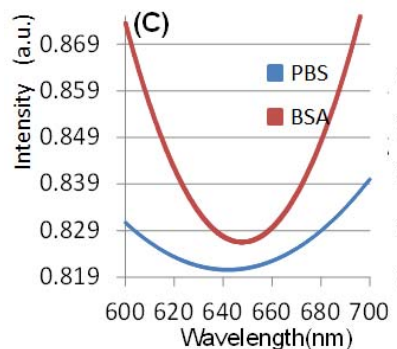

(c)

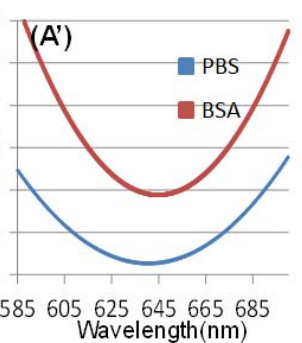

(a')

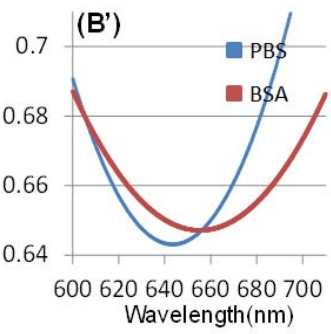

(b')

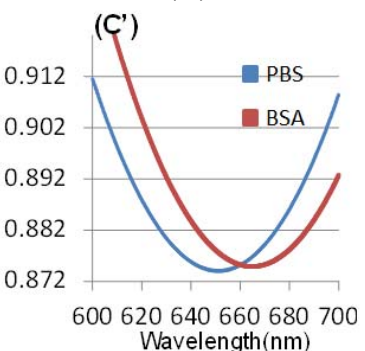

(c')
Fig. 9. LSPR characteristic of changing Au deposition angle ((a) D: $10 \mu \mathrm{m}$ P: $20 \mu \mathrm{m}$, (b) D: $10 \mu \mathrm{m}$ P: $15 \mu \mathrm{m}$, (c) D: $5 \mu \mathrm{m}$ P: $10 \mu \mathrm{m}$ ).

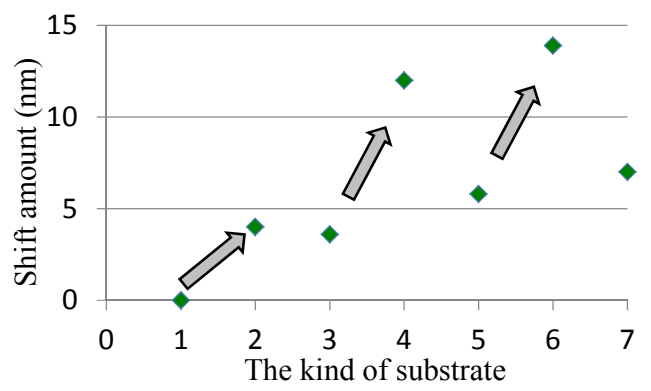

Substrate 1 (D: 10 P:20) $\left(\theta=0^{\circ}\right)$

Substrate 2 (D:10 P:20) $\left(\theta=45^{\circ}\right)$

Substrate 3 (D: 10 P:15) $\left(\theta=0^{\circ}\right)$

Substrate 4 (D: 10 P:15) $\left(\theta=45^{\circ}\right)$

Substrate 5 (D:5 P:10) $\left(\theta=0^{\circ}\right)$

Substrate 6 (D:5 P:10) $\left(\theta=45^{\circ}\right)$

Substrate 7 (D:5 P:7) $\quad\left(\theta=45^{\circ}\right)$

Fig. 10. Shift amount and kinds of substrate.

\section{B. Chang of Irradiation/Detection Angle}

We used patterned CNTs which diameter has5 um, pitch has 10um (Fig. 8(B)) to evaluate influence which changing irradiation/detection angle has on LSPR characteristic.in this experiment, BSA concentration was $100 \mathrm{mg} / \mathrm{l}$.

Fig. 11 shows LSPR characteristic of changing irradiation angle ( $\theta=30,40,45$ degree) before and after BSA adhesion. And, Fig. 12 shows relation between shift amount and substrate.

When the irradiation angle was 30 degrees, the shift amount increased compared with the irradiation angle of 40 degrees, and 45 degrees. Moreover, when the irradiation angle was 40 degrees, the shift amount increased compared with the irradiation angle of 45 degrees.

If the irradiation angle becomes small, the angle of reflected light will also become small. Since the sensor produced in this experiment has three-dimensional structure by CNTs, when the angle of irradiation becomes large, it is for the spot for which reflected light is interrupted by the structure of CNTs to increase

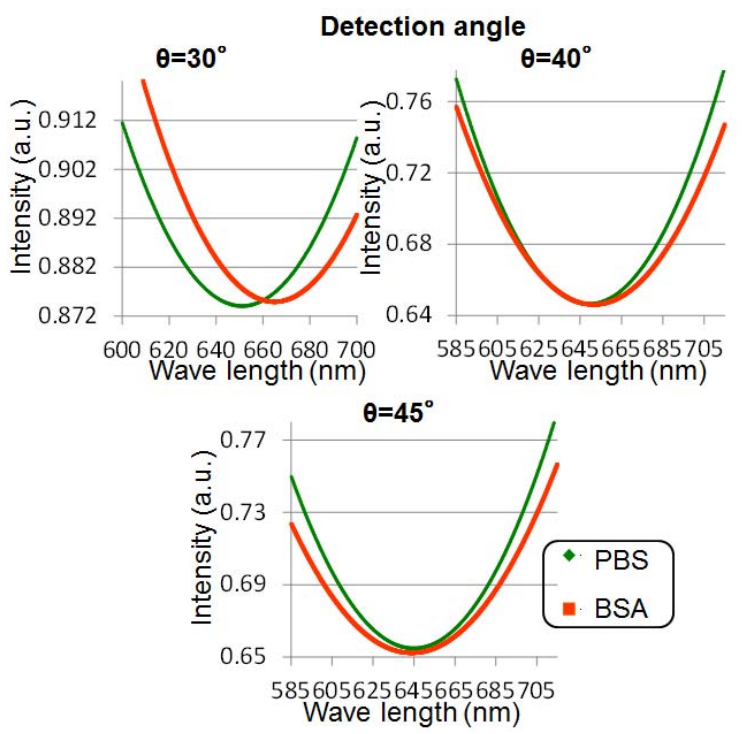

Fig. 11. LSPR characteristic of changing irradiation angle $(\theta=30,40,45$ degree).

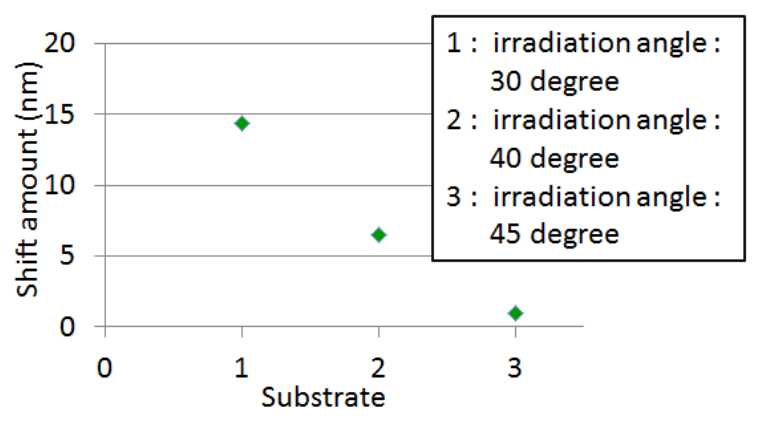

Fig. 12. Relation between shift amount and substrate.

\section{Detection Limit}

We evaluated detection method of this 3-dimentional LSPR sensor. We used BSA as a protein, these concentrations were $1 \mathrm{ng} / \mathrm{ml}, 1,10,100 \mathrm{ug} / \mathrm{ml}$. By using these BSA concentrations, we evaluated LSPR characteristic before and after adherence of BSA, and The relation between a shift amount and concentration was shown.

Fig.13 shows LSPR characteristic of changing BSA 
concentration in patterned CNTs (D: $10 \mu \mathrm{m}$ P: $20 \mu \mathrm{m}$ ). This CNTs length was $10 \mu \mathrm{m}$. In $100 \mu \mathrm{g} / \mathrm{ml}$ of BSA concentration, shift amount was about $7 \mathrm{~nm}$, and shift amount was about $4 \mathrm{~nm}$ in $10 \mu \mathrm{g} / \mathrm{ml}$, and it was about $2 \mathrm{~nm}$ in $1 \mu \mathrm{g} / \mathrm{ml}$, in $1 \mathrm{ng} / \mathrm{ml}$, shift amount was not confirmed. So, in patterned CNTs (D: $10 \mu \mathrm{m}$ P: $20 \mu \mathrm{m}$ Length: $10 \mu \mathrm{m})$, detection limit was $1 \mu \mathrm{g} / \mathrm{ml}$. In future, we have to investigate the detection limit of various patterned $\mathrm{CNTs}$, and it is necessary to optimize structural size of patterned CNTs.

In this experiment, although only CNTs which diameter has 10um and Pitch has 20um estimated the detection limit, so it is necessary to conduct the same experiment in CNTs which has various pitches and a diameter in a future.

Moreover, it is necessary to also make the kind of concentration increase further: for example $1,10,100 \mathrm{ng} / \mathrm{ml}$ Relationship including standard deviation and an error needs to be shown by increasing the number of times of measurement.
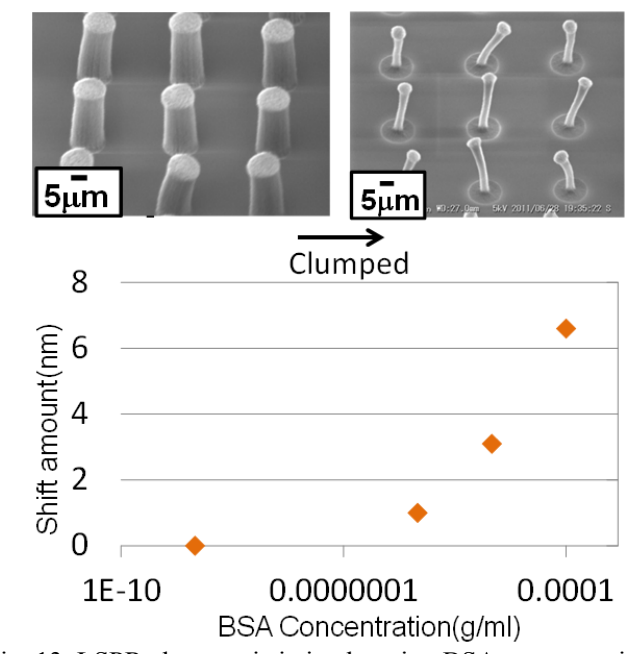

Fig. 13. LSPR characteristic in changing BSA concentration.

\section{CONCLUTION}

In this study, Au nanoparticles were allocated in three dimensions to improve sensitivity than a conventional LSPR sensor, and aim at two points; one is the establishment of a new LSPR sensor and the other is optimal design of detection of low concentration antigen. CNTs was patterned by the lithography, it was seen that wavelength shifted after
BSA adhesion. Therefore, we were able to confirm that the substrate which was allocated metal particles in three dimensions had the function as the sensor. Furthermore, When $\mathrm{Au}$ nanoparticles deposition method changed, the number of Au nanoparticles on CNTs surface increases, so it increased the total absorption of excitation light. Furthermore, by reducing the diameter and pitch, the efficiency of plasmon resonance increased, so shift amount was increased. In addition, in patterned CNTs (D: $10 \mu \mathrm{m}$ P: $20 \mu \mathrm{m})$, detection limit was $1 \mu \mathrm{g} / \mathrm{ml}$. In future, we have to investigate the detection limit of various patterned CNTs, and it is necessary to optimize structural size of patterned CNTs.

\section{REFERENCE}

[1] S. Massenot, "Tunable grating-assisted surface plasmon resonance by use of nano-polymer dispersed liquid crystal electro-optical material,“ Optics Communications, vol.275, pp.318-323,2007

[2] T. R. Jensen, Tunable localized surface plasmon resonance of Silver Spectra of Silver Nanoparticle, 2000

[3] L. J. Sherry, R. Jin, C. A. Mirkin, G. C. Schatz, and R. P. V. Duyne, "Loalized Surface Plasmon Resonance Spectroscopy of Single Silver Triangular Nanoprisms,“ Nano Letters, vol.6, no.9, pp. 2060-2065, 2006

[4] M. Piliarik, M. Vala, I. Tichy, J. Homola, Compact and low-cost biosensor based on novel approach to spectroscopy of surface plasmons, Biosensors and Bioelectronics,2008

[5] S. Basu, S. Pande, S. Jana, S. Bolisetty, T. Pal, Langmuir 24, 5562 2008.

[6] G. H. Jeong, Y.W. Lee, M. Kim, S.W. Han, J. Colloid Interf. Sci. 329 (2009) 97

[7] S. Matsubara, T. Hayakawa, Y. Yang, M. Nogami, S. Okamoto, N. Koshikawa, J. Phys.Chem. C 112, 13917, 2007.

[8] S. Iijima, Nature354, pp.56, 1991

[9] H. Zhang, J. Tang, P. Zhu, J. Ma, L. C. Qin, "High tensile modulus of carbon nano tube nano-fibers produced by dielectrophoresis, “ Chemical Physics Letters 478, pp.230-233, 2009

[10] W. A. De Heer, et al., Since270, p.1179, 1995

[11] J. Okuno, K. Maehashi, K. Matumoto, K. Kerman, Y. Takamura, and E. Tamiya, "Single walled carbon nano tubes-arrayed microelectrode chip for electrochamical analysis, " Electrochemistry Communications 9, pp.13-18, 2007

[12] P. E.lopes , F. V. Hattum, C. M. C. Pereia, P. J. R. O. Novoa, S. Forero, F. Hepp, and L. Pambaguian, "High CNT content composites with CNT buckypaper and epoxy resin matrix:Impregnation behavior composite production and charavcterization," Composite Struture 92, pp.1291-1298, 2010

[13] R. Fujieda, M. Yang, "LSPR Sensitivity Improvement by Using $\mathrm{CNTs} / \mathrm{Au}$ Nanoparticle for Bioanalysis," Advanced Materials Research (Volumes 403 - 408), 4411-4415, 2011 